\title{
Singular Initial Value Problem for Certain Classes of Systems of Ordinary Differential Equations
}

\author{
Josef Diblík, ${ }^{1,2,3}$ Josef Rebenda, ${ }^{3}$ and Zdeněk Šmarda ${ }^{2,3}$ \\ ${ }^{1}$ Department of Mathematics and Descriptive Geometry, Faculty of Civil Engineering, Brno University of Technology, \\ Veverí 331/95, 60200 Brno, Czech Republic \\ ${ }^{2}$ Brno University of Technology, Department of Mathematics, Faculty of Electrical Engineering and Communication, \\ Technická 8, 61600 Brno, Czech Republic \\ ${ }^{3}$ CEITEC BUT, Brno University of Technology, Technická 3058/10, 61600 Brno, Czech Republic
}

Correspondence should be addressed to Josef Diblík; diblik@feec.vutbr.cz

Received 11 August 2013; Accepted 16 October 2013

Academic Editor: Yuriy Rogovchenko

Copyright (C) 2013 Josef Diblík et al. This is an open access article distributed under the Creative Commons Attribution License, which permits unrestricted use, distribution, and reproduction in any medium, provided the original work is properly cited.

\begin{abstract}
The paper is devoted to the study of the solvability of a singular initial value problem for systems of ordinary differential equations. The main results give sufficient conditions for the existence of solutions in the right-hand neighbourhood of a singular point. In addition, the dimension of the set of initial data generating such solutions is estimated. An asymptotic behavior of solutions is determined as well and relevant asymptotic formulas are derived. The method of functions defined implicitly and the topological method (Ważewski's method) are used in the proofs. The results generalize some previous ones on singular initial value problems for differential equations.
\end{abstract}

\section{Introduction}

Let $x_{0}>0$ and $y_{0}>0$ be given constants. Define auxiliary set of points

$$
D_{n}:=\left(0, x_{0}\right] \times \prod_{i=1}^{n}\left(0, y_{0 i}\right]
$$

where $y_{0 i}=y_{0}$.

In the paper, we consider a system of ordinary differential equations in the form

$$
g_{i}(x, y) y_{i}^{\prime}=\alpha_{i}(x, y),
$$

where $i=1, \ldots, n$ and functions $g_{i}, \alpha_{i}: D_{n} \rightarrow(0, \infty)$ can satisfy

$$
g_{r}\left(0^{+}, \theta\right)=\alpha_{r}\left(0^{+}, \theta\right)=0
$$

for some indices $r \in\{1,2, \ldots, n\}$ or

$$
\frac{1}{g_{s}\left(0^{+}, \theta\right)}=\frac{1}{\alpha_{s}\left(0^{+}, \theta\right)}=0
$$

for some indices $s \in\{1,2, \ldots, n\}$, where $\theta=(0,0, \ldots, 0)$ is the $n$-dimensional zero vector. Together with system (2) we consider the initial problem

$$
y_{i}\left(0^{+}\right)=0, \quad i=1, \ldots, n .
$$

Because of the above properties, the initial problem (2), (5) is called a singular initial problem.

Definition 1. Denote by $M\left(x_{0}, y_{0}\right)$ a class of vector-functions $\varphi:\left(0, x_{0}\right] \rightarrow \mathbb{R}^{n}$ having the following properties:

(1) $\varphi$ is continuously differentiable on $\left(0, x_{0}\right]$;

(2) $\varphi_{i}\left(0^{+}\right)=0, i=1, \ldots, n$;

(3) $0<\varphi_{i}^{(j)}(x)$ for $x \in\left(0, x_{0}\right], j=0,1, i=1, \ldots, n$;

(4) $\varphi_{i}\left(x_{0}\right)<y_{0}, i=1, \ldots, n$.

For $\varphi \in M\left(x_{0}, y_{0}\right)$, define an auxiliary vector-function

$$
G(x, \varphi(x))=\left(G_{1}(x, \varphi(x)), \ldots, G_{n}(x, \varphi(x))\right),
$$


where

$$
\begin{array}{r}
G_{i}(x, \varphi(x)):=-\varphi_{i}^{\prime}(x) g_{i}(x, \varphi(x))+\alpha_{i}(x, \varphi(x)), \\
i=1, \ldots, n .
\end{array}
$$

In the paper, sufficient conditions which guarantee the existence of a parametric class of solutions of initial value problem (2), (5) are given and asymptotic formulas

$$
y_{i}(x)=\varphi_{i}(x)(1+o(1)), \quad x \longrightarrow 0^{+}, i=1, \ldots, n
$$

are derived, where $\varphi_{i}$ are the coordinates of a function $\varphi \in$ $M\left(x_{0}, y_{0}\right)$ and the symbol $o(1)$ is the well-known Landau order symbol.

There are numerous papers and books dealing with singular initial value problems (see, e.g., [1-16] and the references therein). Among others, we should mention pioneering results on the solvability of singular problems for ordinary differential equations achieved by Chechyk [15] and Kiguradze [13]. The results of the paper generalize previous investigation of the first author on the solvability of singular problems [5-7]. The main differences are as follows. In [5], a scalar singular differential equation was studied for the case that a function similar to the function $G$ above does not change the sign for $x \rightarrow 0^{+}$. In [6], system (2) is investigated under the assumption that the $i$ th right-hand side of the system is bounded by the product of two functions, with the first depending only on the variable $x$ while the second one only depends on the variable $y_{i}, i=1, \ldots, n$. In comparison with the results of [7], we cannot expect that a first approximation of system (2) consists of equations with separable variables.

The structure of the paper is the following. In Section 2, auxiliary results on implicit functions are given. We refer to Corollary 4 where formula (27) is crucial for the proofs of the asymptotic behavior of solutions. The main results of the paper are formulated in Section 3. New results are proved and a progress is achieved by implicit construction of funnels, where solutions of the singular problem are expected. To prove the existence of such solutions, the topological method of Ważewski (see, e.g., [17-19]) is used. A simple illustrative example is shown here as well. A generalization of the results derived is discussed in Section 4.

\section{Auxiliary Results on Implicit Functions}

First, we give some properties of implicit functions used in the following proofs.

Lemma 2. Assume that a function $\omega: D_{1} \rightarrow \mathbb{R}$ satisfies the following conditions:

(1) $\omega(x, y)$ is continuously differentiable with respect to $x$ and $y$;

(2) $\omega\left(0^{+}, 0^{+}\right)=0$;

(3) for every $y \in\left(0, y_{0}\right]$, there exists a finite limit $\omega\left(0^{+}, y\right)$, for every $x \in\left(0, x_{0}\right]$, there exists a finite limit $\omega\left(x, 0^{+}\right)$, and $\omega\left(0^{+}, y\right) \omega\left(x, 0^{+}\right)<0$;
(4) $\omega_{x}^{\prime}(x, y) \omega_{y}^{\prime}(x, y)<0$, where $(x, y) \in D_{1}$.

Then,

$$
\omega(x, y)=0
$$

defines a unique implicit function $y=y(x)$ on some interval $\left(0, x_{00}\right], 0<x_{00} \leq x_{0}$ such that $y(x) \in M\left(x_{00}, y_{0}\right)$.

Proof. Analysing assumptions (1)-(4), we deduce that only the following two cases can occur: either

$$
\begin{array}{ll}
\omega\left(0^{+}, y\right)<0, & \omega_{y}^{\prime}(x, y)<0, \\
\omega\left(x, 0^{+}\right)>0, & \omega_{x}^{\prime}(x, y)>0
\end{array}
$$

or

$$
\begin{array}{ll}
\omega\left(0^{+}, y\right)>0, & \omega_{y}^{\prime}(x, y)>0, \\
\omega\left(x, 0^{+}\right)<0, & \omega_{x}^{\prime}(x, y)<0
\end{array}
$$

while the remaining two cases

$$
\begin{gathered}
\omega\left(0^{+}, y\right)<0, \quad \omega_{y}^{\prime}(x, y)>0, \quad \omega\left(x, 0^{+}\right)>0, \\
\omega_{x}^{\prime}(x, y)<0, \quad \omega\left(0^{+}, y\right)>0, \quad \omega_{y}^{\prime}(x, y)<0, \\
\omega\left(x, 0^{+}\right)<0, \quad \omega_{x}^{\prime}(x, y)>0
\end{gathered}
$$

are in contradiction with assumptions (1) and (2). The rest of the proof is analogous to the proofs of the wellknown implicit-function theorems and, therefore, we leave it out.

To formulate the second lemma, we need some auxiliary notions. Define, for a given $y_{00}>0$ and $\varepsilon^{*}>0$ satisfying the inequality $0<y_{00}\left(1+\varepsilon^{*}\right)<y_{0}$, the set

$$
D^{*}\left(y_{00}, \varepsilon^{*}\right):=\left\{(y, \varepsilon): y \in\left[0, y_{00}\right], \varepsilon \in\left(-\varepsilon^{*}, \varepsilon^{*}\right)\right\} .
$$

Moreover, for a given continuously differentiable function $R$ : $\left(0, y_{0}\right) \rightarrow(0, \infty)$, let

$$
F(y, \varepsilon):=\frac{R(y(1+\varepsilon))}{R(y)},
$$

where argument $y(1+\varepsilon)$ is assumed to be positive. In addition, we define

$$
\begin{aligned}
& F(0, \varepsilon):=\lim _{y \rightarrow 0^{+}} F(y, \varepsilon), \\
& F_{\varepsilon}^{\prime}(0, \varepsilon):=\lim _{y \rightarrow 0^{+}} F_{\varepsilon}^{\prime}(y, \varepsilon)
\end{aligned}
$$

provided that the limits exist and are finite.

Lemma 3. Assume that functions

$$
R:\left(0, y_{0}\right) \longrightarrow(0, \infty), \quad F: D^{*}\left(y_{00}, \varepsilon^{*}\right) \longrightarrow[0, \infty)
$$

satisfy the following conditions: 
(1) $R(y)$ is continuously differentiable and $R^{\prime}(y)<0$;

(2) $F(y, \varepsilon)$ is continuous with respect to $y$ and continuously differentiable with respect to $\varepsilon$;

(3) $F_{\varepsilon}^{\prime}(y, 0) \neq 0$.

Then, for an arbitrary $y \in\left[0, y_{00}\right]$ and $\varepsilon_{1} \in\left(-\widetilde{\varepsilon}_{1}, \widetilde{\varepsilon}_{1}\right)$, where $\widetilde{\varepsilon}_{1}$ is a positive and sufficiently small constant, there exists a unique continuous solution

$$
\varepsilon_{1}^{0}=\varepsilon_{1}^{0}\left(y, \varepsilon_{1}\right)
$$

of the equation

$$
1+\varepsilon_{1}-F\left(y, \varepsilon_{1}^{0}\right)=0
$$

where

$$
\begin{aligned}
\varepsilon_{1}^{0}\left(y, \varepsilon_{1}\right): & {\left[0, y_{00}\right] \times\left(-\widetilde{\varepsilon}_{1}, \widetilde{\varepsilon}_{1}\right) } \\
\longrightarrow & \begin{cases}\left(-\widetilde{\varepsilon}_{1}^{0}, 0\right) & \text { if } \varepsilon_{1} \in\left(0, \widetilde{\varepsilon}_{1}\right), \\
0 & \text { if } \varepsilon_{1}=0, \\
\left(0, \widetilde{\varepsilon}_{1}^{0}\right) & \text { if } \varepsilon_{1} \in\left(-\widetilde{\varepsilon}_{1}, 0\right),\end{cases}
\end{aligned}
$$

and $\widetilde{\varepsilon}_{1}^{0}$ is a sufficiently small positive constant, $\widetilde{\varepsilon}_{1}^{0} \leq \varepsilon^{*}$.

Proof. Define an auxiliary function

$$
\omega: D^{*}\left(y_{00}, \varepsilon^{*}\right) \times\left(-\widetilde{\varepsilon}_{1}, \widetilde{\varepsilon}_{1}\right) \longrightarrow \mathbb{R}
$$

as

$$
\omega\left(y, \varepsilon_{1}^{0}, \varepsilon_{1}\right):=1+\varepsilon_{1}-F\left(y, \varepsilon_{1}^{0}\right)
$$

and consider implicit equation (18) in the form

$$
\omega\left(y, \varepsilon_{1}^{0}, \varepsilon_{1}\right)=0
$$

with respect to $\varepsilon_{1}^{0}$. In what follows, we will assume that $y \in$ $\left[0, y_{00}\right]$ is a parameter. Since

$$
\omega(y, 0,0)=0,
$$

$\omega\left(y, \varepsilon_{1}^{0}, \varepsilon_{1}\right)$ is continuous with respect to all $y, \varepsilon_{1}^{0}$, and $\varepsilon_{1}$ and continuously differentiable with respect to $\varepsilon_{1}^{0}$, and

$$
\omega_{\varepsilon_{1}^{0}}^{\prime}(y, 0,0)=-F_{\varepsilon_{1}^{0}}^{\prime}(y, 0)>0
$$

for arbitrary $y \in\left[0, y_{00}\right]$. Hence we can apply the classical implicit-function theorem. As a result, we state that (18) is uniquely solvable with respect to $\varepsilon_{1}^{0}$. Thus

$$
\varepsilon_{1}^{0}=\varepsilon_{1}^{0}\left(y, \varepsilon_{1}\right),
$$

where $\varepsilon_{1}^{0}:\left[0, y_{00}\right] \times\left(-\widetilde{\varepsilon}_{1}, \widetilde{\varepsilon}_{1}\right) \rightarrow \mathbb{R}$ is a continuous function with respect to both $y$ and $\varepsilon_{1}$ and $\widetilde{\varepsilon}_{1}$ is a sufficiently small positive number. The sign of the function $\varepsilon_{1}^{0}\left(y, \varepsilon_{1}\right)$ can be specified. In particular, since $R$ is a decreasing function, the function $F$ is decreasing with respect to $\varepsilon$,

$$
\begin{aligned}
\varepsilon_{1}^{0}=\varepsilon_{1}^{0}\left(y, \varepsilon_{1}\right):\left[0, y_{00}\right] \times\left(-\widetilde{\varepsilon}_{1}, \widetilde{\varepsilon}_{1}\right) \\
\quad \longrightarrow \begin{cases}\left(-\widetilde{\varepsilon}_{1}^{0}, 0\right) & \text { if } \varepsilon_{1} \in\left(0, \widetilde{\varepsilon}_{1}\right), \\
0 & \text { if } \varepsilon_{1}=0, \\
\left(0, \widetilde{\varepsilon}_{1}^{0}\right) & \text { if } \varepsilon_{1} \in\left(-\widetilde{\varepsilon}_{1}, 0\right),\end{cases}
\end{aligned}
$$

and $\widetilde{\varepsilon}_{1}^{0}$ is a sufficiently small positive constant satisfying $\widetilde{\varepsilon}_{1}^{0} \leq$ $\varepsilon^{*}$.

Corollary 4. It is possible to reformulate the statement of Lemma 3 as follows. Since (18) is uniquely solvable, one can use the definition of $F(y, \varepsilon)$ given by (14) to get

$$
R(y)\left(1+\varepsilon_{1}\right) \equiv R\left(y\left(1-\varepsilon_{1}^{00}\left(y, \varepsilon_{1}\right)\right)\right),
$$

where $\varepsilon_{1}^{00}\left(y, \varepsilon_{1}\right):=-\varepsilon_{1}^{0}\left(y, \varepsilon_{1}\right)$.

\section{Main Results}

In this part, the main results related to the solvability of problem (2), (5) are proved. We will discuss the dimension of the set of initial conditions generating solutions of this problem as well.

Using $\varphi \in M\left(x_{0}, y_{0}\right)$, define the sets

$$
\begin{gathered}
N^{\varphi_{i}}:=\left\{(x, y):(x, y) \in D_{n}, \varphi_{i}(x)<y_{i}<\varphi_{i}\left(x_{0}\right)\right\}, \\
N_{\varphi_{i}}:=\left\{(x, y):(x, y) \in D_{n}, y_{i}<\varphi_{i}(x)\right\},
\end{gathered}
$$

where $i=1,2, \ldots, n$. To formulate the results we need auxiliary functions

$$
W_{i}: N^{\varphi_{i}} \cup N_{\varphi_{i}} \longrightarrow \mathbb{R}^{n}
$$

defined as follows:

$$
\begin{aligned}
W_{i}(x, y):= & g_{i}\left(\varphi_{i}^{-1}\left(y_{i}\right), \varphi\left(\varphi_{i}^{-1}\left(y_{i}\right)\right)\right) \frac{\alpha_{i}(x, y)}{g_{i}(x, y)} \\
& -\alpha_{i}(x, \varphi(x))
\end{aligned}
$$

for $i=1,2, \ldots, n$.

Theorem 5. Let $g_{i}: D_{n} \rightarrow \mathbb{R}^{n}$ and $\alpha_{i}: D_{n} \rightarrow \mathbb{R}^{n}, i=$ $1, \ldots, n$, be continuous functions. Let, moreover, for a function $\varphi \in M\left(x_{0}, y_{0}\right)$, the following conditions be true:

(1)

$$
\begin{array}{r}
0<g_{i}(x, \varphi(x)), \quad 0<\alpha_{i}(x, \varphi(x)), x \in\left(0, x_{0}\right], \\
i=1, \ldots, n ;
\end{array}
$$

(2)

$$
\begin{array}{r}
\int_{0^{+}} g_{i}(x, \varphi(x)) \varphi_{i}^{\prime}(x) d x=\int_{0^{+}} \alpha_{i}(x, \varphi(x)) d x=\infty, \\
i=1, \ldots, n ;
\end{array}
$$

$$
\left|\int_{x}^{x_{00}} G_{i}(t, \varphi(t)) d t\right|<\left|\int_{x_{k i}}^{x_{00}} g_{i}(t, \varphi(t)) \varphi_{i}^{\prime}(t) d t\right|,
$$

where $x_{00} \in\left(0, x_{0}\right)$ is a sufficiently small constant, $x \in$ $\left(0, x_{00}\right], x_{k i}=\varphi_{i}^{-1}\left(\psi_{k i}\right)$, and $\psi_{k i}, k=1,2, i=1, \ldots, n$, are constants such that

$$
\psi_{1 i}<\varphi_{i}\left(x_{00}\right)<\psi_{2 i}<y_{0}, \quad x_{2 i}<x_{0} ;
$$

(4) there is an integer $n_{1} \in\{0,1, \ldots, n\}$ such that 
(a) $W_{i}(x, y)>0$ if $(x, y) \in N^{\varphi_{i}}$ and $i=1, \ldots, n_{1}$;

(b) $W_{i}(x, y)<0$ if $(x, y) \in N_{\varphi_{i}}$ and $i=1, \ldots, n_{1}$;

(c) $W_{i}(x, y)<0$ if $(x, y) \in N^{\varphi_{i}}$ and $i=n_{1}+1, \ldots, n$;

(d) $W_{i}(x, y)>0$ if $(x, y) \in N_{\varphi_{i}}$ and $i=n_{1}+1, \ldots, n$.

Here, if $n_{1}=0$, conditions (a), (b) are omitted and, if $n_{1}=$ $n$, conditions $(\mathrm{c}),(\mathrm{d})$ are omitted.

Then, problem (2), (5) has at least $n_{1}$-parametric class of solutions $y(x)=\left(y_{1}(x), \ldots, y_{n}(x)\right)$ such that $(x, y(x)) \in D_{n}$ for $x \rightarrow 0^{+}$.

Proof. The proof is divided into two parts. First, implicit curves are constructed and their properties are derived. Then, Ważewski's method is applied to special domains having the shape of funnels with sides constructed using implicitly defined hypersurfaces. In this construction, we use implicit curves from the first part of the proof.

Implicitly Defined Curves and Their Properties. Let $\varphi \in$ $M\left(x_{0}, y_{0}\right)$ be fixed. Define auxiliary functions

$$
z_{k i}, \widetilde{z}_{k i}:\left(0, x_{00}\right] \times\left(0, y_{0}\right] \longrightarrow \mathbb{R}, \quad k=1,2, i=1, \ldots, n
$$

as

$$
\begin{aligned}
z_{k i}\left(x, y_{k i}\right):= & \int_{\psi_{k i}}^{y_{k i}} g_{i}\left(\varphi_{i}^{-1}(t), \varphi\left(\varphi_{i}^{-1}(t)\right)\right) d t \\
& -\int_{x_{00}}^{x} \alpha_{i}(t, \varphi(t)) d t \\
\widetilde{z}_{k i}\left(x, y_{k i}\right):= & {\left[\int_{\psi_{k i}}^{y_{k i}} g_{i}\left(\varphi_{i}^{-1}(t), \varphi\left(\varphi_{i}^{-1}(t)\right)\right) d t\right]^{-1} } \\
& -\left[\int_{x_{00}}^{x} \alpha_{i}(t, \varphi(t)) d t\right]^{-1} .
\end{aligned}
$$

We prove that

$$
z_{k i}\left(x, y_{k i}\right)=0, \quad k=1,2, i=1, \ldots, n,
$$

define unique implicit functions

$$
y_{k i}=y_{k i}(x) \equiv \psi_{k i}(x) \in M\left(x_{00}, y_{0}\right)
$$

on the interval $\left(0, x_{00}\right]$. Observe that the function $\psi_{k i}(x)$ is a solution of

$$
\widetilde{z}_{k i}\left(x, y_{k i}\right)=0, \quad k=1,2, i=1, \ldots, n,
$$

on the interval $\left(0, x_{00}\right)$ as well. Therefore, we consider the latter equation and investigate its solvability using Lemma 2. Set

$$
\omega(x, y):=\widetilde{z}_{k i}(x, y)
$$

where $k \in\{1,2\}$ and $i \in\{1, \ldots, n\}$. We show that the function $\omega(x, y)$ satisfies all assumptions (1)-(4) of Lemma 2, where, instead of the region $D_{1}$, we assume the region $\left(0, x_{00}^{*}\right] \times$ $\left(0, y_{0}^{*}\right]$ with sufficiently small $x_{00}^{*}, y_{0}^{*}$ such that $0<x_{00}^{*}<x_{00}$, $0<y_{0}^{*}<y_{0}$.

(a) It is easy to see (in view of the above assumptions) that the function

$$
\omega:\left(0, x_{00}^{*}\right] \times\left(0, y_{0}^{*}\right] \longrightarrow \mathbb{R}
$$

defined by (39) is continuously differentiable with respect to $x$ and $y$ and assumption (1) of Lemma 2, holds.

(b) Compute the limit $\omega\left(0^{+}, 0^{+}\right)$. We get

$$
\begin{aligned}
\omega\left(0^{+}, 0^{+}\right) & \\
\equiv & \widetilde{z}_{k i}\left(0^{+}, 0^{+}\right) \\
= & \lim _{x \rightarrow 0^{+}, y \rightarrow 0^{+}}\left[\left[\int_{\psi_{k i}}^{y} g_{i}\left(\varphi_{i}^{-1}(t), \varphi\left(\varphi_{i}^{-1}(t)\right)\right) d t\right]^{-1}\right. \\
= & \left.\left.-\left[\int_{y \rightarrow 0^{+}}^{x} \alpha_{i}(t, \varphi(t)) d t\right]^{-1}\right]_{\psi_{k i}}^{y} g_{i}\left(\varphi_{i}^{-1}(t), \varphi\left(\varphi_{i}^{-1}(t)\right)\right) d t\right]^{-1} \\
& -\lim _{x \rightarrow 0^{+}}\left[\int_{x_{00}}^{x} \alpha_{i}(t, \varphi(t)) d t\right]^{-1}
\end{aligned}
$$

if the last two limits exist and are finite. Substituting $t=$ $\varphi_{i}(x)$ into the first integral of the last expression and using condition (2), we get

$$
\begin{aligned}
\omega\left(0^{+}, 0^{+}\right) \equiv & \widetilde{z}_{k i}\left(0^{+}, 0^{+}\right) \\
= & \lim _{y \rightarrow 0^{+}}\left[\int_{\varphi_{i}^{-1}\left(\psi_{k i}\right)}^{\varphi_{i}^{-1}(y)} g_{i}(x, \varphi(x)) \varphi_{i}^{\prime}(x) d x\right]^{-1} \\
& -\lim _{x \rightarrow+0}\left[\int_{x_{00}}^{x} \alpha_{i}(t, \varphi(t)) d t\right]^{-1} \\
= & {\left[\int_{x_{k i}}^{0^{+}} g_{i}(x, \varphi(x)) \varphi_{i}^{\prime}(x) d x\right]^{-1} } \\
& -\left[\int_{x_{00}}^{0^{+}} \alpha_{i}(x, \varphi(x)) d x\right]^{-1}=0
\end{aligned}
$$

and assumption (2) of Lemma 2 holds. 
(c) Now we consider the existence of the product $\omega\left(0^{+}, y\right) \omega\left(x, 0^{+}\right)$for $y \in\left(0, y_{0}^{*}\right], x \in\left(0, x_{00}^{*}\right]$ and determine its sign. We get

$$
\begin{gathered}
\omega\left(0^{+}, y\right) \omega\left(x, 0^{+}\right) \\
\equiv \widetilde{z}_{k i}\left(0^{+}, y\right) \widetilde{z}_{k i}\left(x, 0^{+}\right) \\
=\lim _{x \rightarrow 0^{+}}\left[\left[\int_{\psi_{k i}}^{y} g_{i}\left(\varphi_{i}^{-1}(t), \varphi\left(\varphi_{i}^{-1}(t)\right)\right) d t\right]^{-1}\right. \\
\left.-\left[\int_{x_{00}}^{x} \alpha_{i}(t, \varphi(t)) d t\right]^{-1}\right] \\
\times \lim _{y \rightarrow 0^{+}}\left[\left[\int_{\psi_{k i}}^{y} g_{i}\left(\varphi_{i}^{-1}(t), \varphi\left(\varphi_{i}^{-1}(t)\right)\right) d t\right]^{-1}\right. \\
\left.-\left[\int_{x_{00}}^{x} \alpha_{i}(t, \varphi(t)) d t\right]^{-1}\right] .
\end{gathered}
$$

Substituting $t=\varphi_{i}(x)$ into the first integrals in the square brackets, using conditions (1) and (2) of Theorem 5 and the property $x_{00}^{*}<x_{00}$, and assuming $y_{0}^{*}<\min _{k=1,2 ; i=1, \ldots, n}\left\{\psi_{k i}\right\}$, we have

$$
\begin{aligned}
\omega\left(0^{+}, y\right) \omega\left(x, 0^{+}\right) \\
\equiv \widetilde{z}_{k i}\left(0^{+}, y\right) \widetilde{z}_{k i}\left(x, 0^{+}\right) \\
=\left[\left[\int_{\varphi_{i}^{-1}\left(\psi_{k i}\right)}^{\varphi_{i}^{-1}(y)} g_{i}(x, \varphi(x)) \varphi_{i}^{\prime}(x) d x\right]^{-1}\right. \\
\left.\quad-\left[\int_{x_{00}}^{0^{+}} \alpha_{i}(x, \varphi(x)) d x\right]^{-1}\right] \\
\times\left[\left[\int_{\varphi_{i}^{-1}\left(\psi_{k i}^{+}\right)}^{0^{+}} g_{i}(x, \varphi(x)) \varphi_{i}^{\prime}(x) d x\right]^{-1}\right. \\
\left.\quad-\left[\int_{x_{00}}^{x} \alpha_{i}(x, \varphi(x)) d x\right]^{-1}\right] \\
=\left[\int_{x_{k i}}^{\varphi_{i}^{-1}(y)} g_{i}(x, \varphi(x)) \varphi_{i}^{\prime}(x) d x\right]^{-1} \\
\quad \times\left[\int_{x_{00}}^{x} \alpha_{i}(x, \varphi(x)) d x\right]^{-1} \cdot(-1)<0 .
\end{aligned}
$$

(d) Determine the sign of $\omega_{x}^{\prime}(x, y) \omega_{y}^{\prime}(x, y)$. We get

$$
\begin{aligned}
& \omega_{x}^{\prime}(x, y) \cdot \omega_{y}^{\prime}(x, y) \\
& \quad \equiv\left(\widetilde{z}_{k i}(x, y)\right)_{x}^{\prime} \cdot\left(\widetilde{z}_{k i}(x, y)\right)_{y}^{\prime}
\end{aligned}
$$

$$
\begin{aligned}
= & (-1) \cdot \alpha_{i}(x, \varphi(x))\left[\int_{x_{00}}^{x} \alpha_{i}(t, \varphi(t)) d t\right]^{-2} \\
& \times\left[\int_{\psi_{k i}}^{y} g_{i}\left(\varphi_{i}^{-1}(t), \varphi\left(\varphi_{i}^{-1}(t)\right)\right) d t\right]^{-2} \\
& \times g_{i}\left(\varphi_{i}^{-1}(y), \varphi\left(\varphi_{i}^{-1}(y)\right)\right) .
\end{aligned}
$$

From condition (1), it follows that

$$
\alpha_{i}(x, \varphi(x))>0, \quad g_{i}\left(\varphi_{i}^{-1}(y), \varphi\left(\varphi_{i}^{-1}(y)\right)\right)>0 .
$$

Hence

$$
\omega_{x}^{\prime}(x, y) \cdot \omega_{y}^{\prime}(x, y) \equiv\left(\widetilde{z}_{k i}(x, y)\right)_{x}^{\prime} \cdot\left(\widetilde{z}_{k i}(x, y)\right)_{y}^{\prime}<0 .
$$

Because of (a)-(d) all assumptions (1)-(4) of Lemma 2 on $\left(0, x_{00}^{*}\right] \times\left(0, y_{0}^{*}\right]$ hold and $(39)$ defines an implicit function

$$
y(x)=y_{k i}(x)=\psi_{k i}(x)
$$

on some interval $\left(0, x_{00}^{* *}\right]$, where $x_{00}^{* *} \leq x_{00}^{*}$.

Now we turn to (37) and show that its solution given by formula (49) can be extended beyond $x_{00}^{* *}$.

We show that $x_{00}^{* *}=x_{00}$. On the contrary, assume $x_{00}^{* *}<$ $x_{00}$. Then, (after a proper transformation of variables) we can apply Lemma 2 to the point $\left(x_{00}^{* *}, \psi_{k i}\left(x_{00}^{* *}\right)\right)$ again and, by well-known procedure, implicit function can be continued up to the boundary of the region $\left(0, x_{00}\right] \times\left(0, y_{0}\right]$.

If $\psi_{k i}\left(x_{00}^{* *}\right)=y_{0}$, then $\widetilde{z}_{k i}\left(x_{00}^{* *}, y_{0}\right)=z_{k i}\left(x_{00}^{* *}, y_{0}\right)=0$. Moreover, we have

$$
\begin{aligned}
z_{k i}\left(x_{00}^{* *}, y_{0}\right)= & \int_{\psi_{k i}}^{y_{0}} g_{i}\left(\varphi_{i}^{-1}(t), \varphi\left(\varphi_{i}^{-1}(t)\right)\right) d t \\
& -\int_{x_{00}}^{x_{00}^{* *}} \alpha_{i}(t, \varphi(t)) d t>0
\end{aligned}
$$

since $x_{00}^{* *}<x_{00}$ and $\psi_{k i}<y_{0}$ by condition (3). This is a contradiction and $x_{00}^{* *}=x_{00}$. Therefore, the implicit function $y_{k i}=\psi_{k i}(x)$ can be continued on the whole interval $\left(0, x_{00}\right]$. Similarly we will show that the inequality

$$
\psi_{k i}\left(x_{00}\right)<y_{0}
$$

holds. We have

$$
\begin{aligned}
z_{k i}\left(x_{00}, y_{0}\right) & \\
= & \int_{\psi_{k i}}^{y_{0}} g_{i}\left(\varphi_{i}^{-1}(t), \varphi\left(\varphi_{i}^{-1}(t)\right)\right) d t \\
& -\int_{x_{00}}^{x_{00}} \alpha_{i}(t, \varphi(t)) d t \\
= & g_{i}\left(\varphi_{i}^{-1}(t), \varphi\left(\varphi_{i}^{-1}(t)\right)\right) d t>0,
\end{aligned}
$$

because, by condition (3), $\psi_{k i}<y_{0}$ holds. It is obvious that

$$
\begin{gathered}
z_{k i}\left(x_{00}, \psi_{k i}\left(x_{00}\right)\right)=0, \\
\left(z_{k i}\left(x_{00}, y\right)\right)_{y}^{\prime}=g_{i}\left(\varphi_{i}^{-1}(y), \varphi\left(\varphi_{i}^{-1}(y)\right)\right)>0 .
\end{gathered}
$$


Because the function $z_{k i}\left(x_{00}, y\right)$ is monotonously increasing and

$$
0=z_{k i}\left(x_{00}, \psi_{k i}\left(x_{00}\right)\right)<z_{k i}\left(x_{00}, y_{0}\right),
$$

we get $\psi_{k i}\left(x_{00}\right)<y_{0}$. Hence inequality (51) is proved.

Now we will investigate the behavior of implicit curves in a neighborhood of the function $\varphi$. Since

$$
\psi_{1 i}<\varphi_{i}\left(x_{00}\right)<\psi_{2 i},
$$

we have (by condition (8))

$$
\begin{gathered}
\varphi_{i}\left(x_{1 i}\right)<\varphi_{i}\left(x_{00}\right)<\varphi_{i}\left(x_{2 i}\right), \\
x_{1 i}<x_{00}<x_{2 i} .
\end{gathered}
$$

Thus, (in the first integral we substitute $t=\varphi_{i}(s)$ )

$$
\begin{aligned}
& z_{k i}\left(x, \varphi_{i}(x)\right) \\
&=\int_{\psi_{k i}}^{\varphi_{i}(x)} g_{i}\left(\varphi_{i}^{-1}(t), \varphi\left(\varphi_{i}^{-1}(t)\right)\right) d t \\
& \quad-\int_{x_{00}}^{x} \alpha_{i}(t, \varphi(t)) d t \\
&=\int_{\varphi_{i}^{-1}\left(\psi_{k i}\right)}^{x} g_{i}(s, \varphi(s)) \varphi_{i}^{\prime}(s) d s \\
& \quad-\int_{x_{00}}^{x} \alpha_{i}(t, \varphi(t)) d t \\
&=\int_{x_{00}}^{x}\left[g_{i}(t, \varphi(t)) \varphi_{i}^{\prime}(t)-\alpha_{i}(t, \varphi(t))\right] d t \\
&+\int_{x_{k i}}^{x_{00}} g_{i}(t, \varphi(t)) \varphi_{i}^{\prime}(t) d t \\
&=-\int_{x_{00}}^{x} G_{i}(t, \varphi(t)) d t \\
&-\int_{x_{00}}^{x_{k i}} g_{i}(t, \varphi(t)) \varphi_{i}^{\prime}(t) d t,
\end{aligned}
$$

where $k=1,2$ and $i=1, \ldots, n$. Consequently, we deduce that

$$
z_{1 i}\left(x, \varphi_{i}(x)\right)>0, \quad z_{2 i}\left(x, \varphi_{i}(x)\right)<0, \quad x \in\left(0, x_{00}\right] .
$$

Since functions $z_{1 i}, z_{2 i}$ increase with respect to their second co-ordinates and

$$
z_{1 i}\left(x, \psi_{1 i}(x)\right) \equiv 0, \quad z_{2 i}\left(x, \psi_{2 i}(x)\right) \equiv 0
$$

on $\left(0, x_{00}\right]$, we get

$$
\psi_{1 i}(x)<\varphi_{i}(x)<\psi_{2 i}(x), \quad x \in\left(0, x_{00}\right]
$$

for each $i \in\{1, \ldots, n\}$. Finally, we recall that

$$
\psi_{k i}\left(0^{+}\right)=\varphi_{i}\left(0^{+}\right)=0, \quad k=1,2, \quad i=1, \ldots, n .
$$

Application of Ważewski's Method to an Implicitly Defined Domain. In the next part of the proof we will apply the topological method of Ważewski. We use the above mentioned functions given implicitly to define an open set

$$
\begin{aligned}
\Omega^{0}:=\left\{(x, y):(x, y) \in D_{n}, v_{k}(x, y)<0,\right. & \\
& \left.\quad k=0, \ldots, n_{1}, u_{j}(x, y)<0, j=n_{1}+1, \ldots, n\right\},
\end{aligned}
$$

where

$$
\begin{array}{r}
v_{0}(x, y) \equiv v_{0}(x):=x-\hat{x}, \quad 0<\hat{x}<x_{00}, \hat{x} \text { is a constant, } \\
v_{k}(x, y) \equiv v_{k}\left(x, y_{k}\right):=\left(y_{k}-\psi_{1 k}(x)\right)\left(y_{k}-\psi_{2 k}(x)\right), \\
k=1, \ldots, n_{1}, \\
u_{j}(x, y) \equiv u_{j}\left(x, y_{j}\right):=\left(y_{j}-\psi_{1 j}(x)\right)\left(y_{j}-\psi_{2 j}(x)\right), \\
j=n_{1}+1, \ldots, n .
\end{array}
$$

Now we start to investigate the behavior of the integral curves of system (2) with respect to the boundary of the set $\Omega^{0}$, that is, on the sets

$$
\begin{array}{r}
V_{\beta}=\left\{(x, y):(x, y) \in D_{n}, v_{\beta}(x, y)=0, v_{k}(x, y) \leq 0,\right. \\
\left.k=0, \ldots, n_{1}, k \neq \beta, u_{j}(x, y) \leq 0, j=n_{1}+1, \ldots, n\right\}, \\
\beta=0, \ldots, n_{1}, \\
U_{\alpha}=\left\{(x, y):(x, y) \in D_{n}, u_{\alpha}(x, y)=0, v_{k}(x, y) \leq 0,\right. \\
\left.k=0, \ldots, n_{1}, u_{j}(x, y) \leq 0, j=n_{1}+1, \ldots, n, j \neq \alpha\right\}, \\
\alpha=n_{1}+1, \ldots, n .
\end{array}
$$

First, we calculate the full derivative $\dot{v}_{\beta}(x, y)$ of the function $v_{\beta}(x, y)$ along trajectories of system (2) on the set $V_{\beta}, \beta=0, \ldots, n_{1}$. It is clear that $\dot{v}_{0}(x)=1>0$. Further, for $\beta=1, \ldots, n_{1}$, we have

$$
\begin{aligned}
\dot{v}_{\beta}(x, y)= & \frac{d}{d x}\left[\left(y_{\beta}-\psi_{1 \beta}(x)\right)\left(y_{\beta}-\psi_{2 \beta}(x)\right)\right] \\
= & {\left[\frac{d}{d x}\left(y_{\beta}-\psi_{1 \beta}(x)\right)\right]\left(y_{\beta}-\psi_{2 \beta}(x)\right) } \\
& +\left(y_{\beta}-\psi_{1 \beta}(x)\right)\left[\frac{d}{d x}\left(y_{\beta}-\psi_{2 \beta}(x)\right)\right] \\
= & {\left[\frac{\alpha_{\beta}(x, y)}{g_{\beta}(x, y)}-\psi_{1 \beta}^{\prime}(x)\right]\left(y_{\beta}-\psi_{2 \beta}(x)\right) } \\
& +\left[\frac{\alpha_{\beta}(x, y)}{g_{\beta}(x, y)}-\psi_{2 \beta}^{\prime}(x)\right]\left(y_{\beta}-\psi_{1 \beta}(x)\right) .
\end{aligned}
$$

On the set $V_{\beta}$, as it follows from the condition $v_{\beta}(x, y)=0$, we have either $y_{\beta}=\psi_{1 \beta}(x)$ or $y_{\beta}=\psi_{2 \beta}(x)$. 
(1) Let $y_{\beta}=\psi_{1 \beta}(x)$. Then, we can see that

$$
\begin{aligned}
\dot{v}_{\beta}\left(x, \psi_{1 \beta}(x)\right) \\
=\left[\frac{\alpha_{\beta}\left(x, y_{1}, \ldots, y_{\beta-1}, \psi_{1 \beta}(x), y_{\beta+1}, \ldots, y_{n}\right)}{g_{\beta}\left(x, y_{1}, \ldots, y_{\beta-1}, \psi_{1 \beta}(x), y_{\beta+1}, \ldots, y_{n}\right)}-\psi_{1 \beta}^{\prime}(x)\right] \\
\quad \times\left(\psi_{1 \beta}(x)-\psi_{2 \beta}(x)\right) .
\end{aligned}
$$

The derivative $\psi_{1 \beta}^{\prime}(x)$ of the function $\psi_{1 \beta}(x)$ can be calculated using the well-known rules for differentiation of implicit functions given by identities

$$
z_{1 \beta}\left(x, \psi_{1 \beta}(x)\right) \equiv 0
$$

We get

$$
\psi_{1 \beta}^{\prime}(x) \equiv \frac{\alpha_{\beta}(x, \varphi(x))}{g_{\beta}\left[\varphi_{\beta}^{-1}\left(\psi_{1 \beta}(x)\right), \varphi\left(\varphi_{\beta}^{-1}\left(\psi_{1 \beta}(x)\right)\right)\right]} .
$$

Using that relation, we have

$$
\begin{aligned}
\dot{v}_{\beta}\left(x, \psi_{1 \beta}(x)\right) & {\left[\frac{\alpha_{\beta}\left(x, y_{1}, \ldots, y_{\beta-1}, \psi_{1 \beta}(x), y_{\beta+1}, \ldots, y_{n}\right)}{g_{\beta}\left(x, y_{1}, \ldots, y_{\beta-1}, \psi_{1 \beta}(x), y_{\beta+1}, \ldots, y_{n}\right)}\right.} \\
& \left.+(-1) \frac{\alpha_{\beta}(x, \varphi(x))}{g_{\beta}\left[\varphi_{\beta}^{-1}\left(\psi_{1 \beta}(x)\right), \varphi\left(\varphi_{\beta}^{-1}\left(\psi_{1 \beta}(x)\right)\right)\right]}\right] \\
& \times\left(\psi_{1 \beta}(x)-\psi_{2 \beta}(x)\right) \\
g_{\beta}\left[\varphi_{\beta}^{-1}\left(\psi_{1 \beta}(x)\right), \varphi\left(\varphi_{\beta}^{-1}\left(\psi_{1 \beta}(x)\right)\right)\right] & {\left[g_{1 \beta}\left[\varphi_{\beta}^{-1}\left(\psi_{1 \beta}(x)\right), \varphi\left(\varphi_{\beta}^{-1}\left(\psi_{1 \beta}(x)\right)\right)\right]\right.} \\
& \quad \times \frac{\alpha_{\beta}\left(x, y_{1}, \ldots, y_{\beta-1}, \psi_{1 \beta}(x), y_{\beta+1}, \ldots, y_{n}\right)}{g_{\beta}\left(x, y_{1}, \ldots, y_{\beta-1}, \psi_{1 \beta}(x), y_{\beta+1}, \ldots, y_{n}\right)} \\
& \left.\quad-\alpha_{\beta}(x, \varphi(x))\right] .
\end{aligned}
$$

Since, by $(60), \psi_{1 \beta}(x)<\psi_{2 \beta}(x)$ and $\psi_{1 \beta}(x)<\varphi_{\beta}(x)<\psi_{2 \beta}(x)$, assumption (1) of the theorem yields

$$
g_{\beta}\left[\varphi_{\beta}^{-1}\left(\psi_{1 \beta}(x)\right), \varphi\left(\varphi_{\beta}^{-1}\left(\psi_{1 \beta}(x)\right)\right)\right]>0 .
$$

In view of assumption (4b) $\left(W_{\beta}(x, y)<0\right.$ when $\left.(x, y) \in N_{\varphi_{\beta}}\right)$, we get

$$
\begin{aligned}
g_{\beta}\left[\varphi_{\beta}^{-1}\left(\psi_{1 \beta}(x)\right), \varphi\left(\varphi_{\beta}^{-1}\left(\psi_{1 \beta}(x)\right)\right)\right] \\
\quad \times \frac{\alpha_{\beta}\left(x, y_{1}, \ldots, y_{\beta-1}, \psi_{1 \beta}(x), y_{\beta+1}, \ldots, y_{n}\right)}{g_{\beta}\left(x, y_{1}, \ldots, y_{\beta-1}, \psi_{1 \beta}(x), y_{\beta+1}, \ldots, y_{n}\right)} \\
-\alpha_{\beta}(x, \varphi(x))<0
\end{aligned}
$$

and, consequently,

$$
\dot{v}_{\beta}\left(x, \psi_{1 \beta}(x)\right)>0 \text {. }
$$

(2) Let $y_{\beta}=\psi_{2 \beta}(x)$. Then, by similar calculations and using assumption (4a) $\left(W_{\beta}(x, y)>0\right.$ when $\left.(x, y) \in N^{\varphi_{\beta}}\right)$, we obtain

$$
\dot{v}_{\beta}\left(x, \psi_{2 \beta}(x)\right)>0
$$

Hence $\dot{v}_{\beta}(x, y)>0$ for all $\beta=1, \ldots, n_{1}$.

Now we will calculate the full derivative $\dot{u}_{\alpha}(x, y)$ of the function $u_{\alpha}(x, y)$ along trajectories of system (2) on the set $U_{\alpha}$, where $\alpha=n_{1}+1, \ldots, n$. As above, we get

$$
\begin{aligned}
\dot{u}_{\alpha}(x, y)= & \frac{d}{d x}\left[\left(y_{\alpha}-\psi_{1 \alpha}(x)\right)\left(y_{\alpha}-\psi_{2 \alpha}(x)\right)\right] \\
= & {\left[\frac{d}{d x}\left(y_{\alpha}-\psi_{1 \alpha}(x)\right)\right]\left(y_{\alpha}-\psi_{2 \alpha}(x)\right) } \\
& +\left(y_{\alpha}-\psi_{1 \alpha}(x)\right)\left[\frac{d}{d x}\left(y_{\alpha}-\psi_{2 \alpha}(x)\right)\right] \\
= & {\left[\frac{\alpha_{\alpha}(x, y)}{g_{\alpha}(x, y)}-\psi_{1 \alpha}^{\prime}(x)\right]\left(y_{\alpha}-\psi_{2 \alpha}(x)\right) } \\
& +\left[\frac{\alpha_{\alpha}(x, y)}{g_{\alpha}(x, y)}-\psi_{2 \alpha}^{\prime}(x)\right]\left(y_{\alpha}-\psi_{1 \alpha}(x)\right) .
\end{aligned}
$$

On the set $U_{\alpha}$, as it follows from the condition $u_{\alpha}(x, y)=0$, we have either $y_{\alpha}=\psi_{1 \alpha}(x)$ or $y_{\alpha}=\psi_{2 \alpha}(x)$.

(1) Let $y_{\alpha}=\psi_{1 \alpha}(x)$. Then, we get (proceeding like in the previous part of the proof)

$$
\begin{aligned}
\dot{u}_{\alpha}\left(x, \psi_{1 \alpha}(x)\right) & \\
= & {\left[\frac{\alpha_{\alpha}\left(x, y_{1}, \ldots, y_{\alpha-1}, \psi_{1 \alpha}(x), y_{\alpha+1}, \ldots, y_{n}\right)}{g_{\alpha}\left(x, y_{1}, \ldots, y_{\alpha-1}, \psi_{1 \alpha}(x), y_{\alpha+1}, \ldots, y_{n}\right)}\right.} \\
& \left.\quad+(-1) \frac{\alpha_{\alpha}(x, \varphi(x))}{g_{\alpha}\left[\varphi_{\alpha}^{-1}\left(\psi_{1 \alpha}(x)\right), \varphi\left(\varphi_{\alpha}^{-1}\left(\psi_{1 \alpha}(x)\right)\right)\right]}\right] \\
& \quad \times\left(\psi_{1 \alpha}(x)-\psi_{2 \alpha}(x)\right)
\end{aligned}
$$




$$
\begin{aligned}
& =\frac{\psi_{1 \alpha}(x)-\psi_{2 \alpha}(x)}{g_{\alpha}\left[\varphi_{\alpha}^{-1}\left(\psi_{1 \alpha}(x)\right), \varphi\left(\varphi_{\alpha}^{-1}\left(\psi_{1 \alpha}(x)\right)\right)\right]} \\
& \times\left[g_{\alpha}\left[\varphi_{\alpha}^{-1}\left(\psi_{1 \alpha}(x)\right), \varphi\left(\varphi_{\alpha}^{-1}\left(\psi_{1 \alpha}(x)\right)\right)\right]\right. \\
& \quad \times \frac{\alpha_{\alpha}\left(x, y_{1}, \ldots, y_{\alpha-1}, \psi_{1 \alpha}(x), y_{\alpha+1}, \ldots, y_{n}\right)}{g_{\alpha}\left(x, y_{1}, \ldots, y_{\alpha-1}, \psi_{1 \alpha}(x), y_{\alpha+1}, \ldots, y_{n}\right)} \\
& \left.\quad-\alpha_{\alpha}(x, \varphi(x))\right] .
\end{aligned}
$$

Since, by $(60), \psi_{1 \alpha}(x)<\psi_{1 \beta}(x)$ and $\psi_{1 \alpha}(x)<\varphi_{\alpha}(x)<$ $\psi_{2 \alpha}(x)$, applying assumption (1) of the theorem, we have

$$
g_{\alpha}\left[\varphi_{\alpha}^{-1}\left(\psi_{1 \alpha}(x)\right), \varphi\left(\varphi_{\alpha}^{-1}\left(\psi_{1 \alpha}(x)\right)\right)\right]>0
$$

In view of assumption $(4 \mathrm{~d})\left(W_{\alpha}(x, y)>0\right.$ when $\left.(x, y) \in N_{\varphi_{\alpha}}\right)$, we have

$$
\begin{aligned}
g_{\alpha}\left[\varphi_{\alpha}^{-1}\left(\psi_{1 \alpha}(x)\right), \varphi\left(\varphi_{\alpha}^{-1}\left(\psi_{1 \alpha}(x)\right)\right)\right] \\
\quad \times \frac{\alpha_{\alpha}\left(x, y_{1}, \ldots, y_{\alpha-1}, \psi_{1 \alpha}(x), y_{\alpha+1}, \ldots, y_{n}\right)}{g_{\alpha}\left(x, y_{1}, \ldots, y_{\alpha-1}, \psi_{1 \alpha}(x), y_{\alpha+1}, \ldots, y_{n}\right)} \\
-\alpha_{\alpha}(x, \varphi(x))>0
\end{aligned}
$$

and, consequently

$$
\dot{u}_{\alpha}\left(x, \psi_{1 \alpha}(x)\right)<0 .
$$

(2) Let $y_{\alpha}=\psi_{2 \alpha}(x)$. Then, by similar calculations and using assumption (4)-(c) $\left(W_{\alpha}(x, y)<0\right.$ when $\left.(x, y) \in N^{\varphi_{\alpha}}\right)$, we obtain

$$
\dot{u}_{\alpha}\left(x, \psi_{2 \alpha}(x)\right)<0 .
$$

Thus, $\dot{u}_{\alpha}(x, y)<0$ for all $\alpha=n_{1}+1, \ldots, n$.

By [18, Lemma 3.1, page 281], for decreasing values of the variable $x$, the set of all egress points $\Omega_{e}^{0}, \Omega_{e}^{0} \subset \Omega^{0}$, from the set $\Omega^{0}$ equals the set of all strict egress points $\Omega_{\mathrm{se}}^{0}, \Omega_{\mathrm{se}}^{0} \subset \Omega^{0}$ from the set $\Omega^{0}$ (for definitions of $\Omega_{e}^{0}$ and $\Omega_{\text {se }}^{0}$ see, for example, [18, page 37 and page 278$]$ ); that is,

$$
\Omega_{e}^{0}=\Omega_{\mathrm{se}}^{0}=\bigcup_{\alpha=n_{1}+1}^{n} U_{\alpha} \backslash \bigcup_{\beta=0}^{n_{1}} V_{\beta} .
$$

Let $S$ be a subset of $\Omega^{0} \cup \Omega_{e}^{0}$ defined as

$$
\begin{gathered}
S=\left\{\left(\tilde{x}, y_{1}^{0}, \ldots, y_{n_{1}}^{0}, y_{n_{1}+1}, \ldots, y_{n}\right): u_{j}\left(\tilde{x}, y_{j}\right) \leq 0,\right. \\
j=n_{1}+1, \ldots, n, v_{0}(\tilde{x})<0, v_{k}\left(\tilde{x}, y_{k}^{0}\right)<0, \\
\left.k=1, \ldots, n_{1}\right\},
\end{gathered}
$$

where $y_{k}^{0}, k=1, \ldots, n_{1}$, are fixed. Then,

$$
\begin{gathered}
S \cap \Omega_{e}^{0}=\left\{\left(\tilde{x}, y_{1}^{0}, \ldots, y_{n_{1}}^{0}, y_{n_{1}+1}, \ldots, y_{n}\right): u_{\alpha}\left(\tilde{x}, y_{\alpha}\right)\right. \\
=0, u_{j}\left(\tilde{x}, y_{j}\right) \leq 0, \alpha, j=n_{1}+1, \ldots, n, \\
\left.\alpha \neq j, v_{k}\left(\tilde{x}, y_{k}^{0}\right)<0, k=1, \ldots, n_{1}\right\} .
\end{gathered}
$$

We can see that the set $S \cap \Omega_{e}^{0}$ is a subset of the boundary $\partial S$ of the set $S$, but it is not a retract of $S$. The explanation is simple and is based on the well-known fact that the boundary of an $\left(n-n_{1}\right)$-dimensional ball is not its retract [20], and the set $S$ is topologically equivalent to an $\left(n-n_{1}\right)$-dimensional ball.

We show that $S \cap \Omega_{e}^{0}$ is a retract of $\Omega_{e}^{0}$. Define a mapping

$$
\pi: \Omega_{e}^{0} \ni(x, y) \longmapsto\left(\tilde{x}, y_{1}^{0}, \ldots, y_{n_{1}}^{0}, \tilde{y}_{n_{1}+1}, \ldots, \tilde{y}_{n}\right) \in S \cap \Omega_{e}^{0} \text {, }
$$

where

$$
\begin{aligned}
& \tilde{y}_{j}=\psi_{1 j}(\tilde{x})+\left(y_{j}-\psi_{1 j}(x)\right) \frac{\psi_{2 j}(\tilde{x})-\psi_{1 j}(\tilde{x})}{\psi_{2 j}(x)-\psi_{1 j}(x)}, \\
& j=n_{1}+1, \ldots, n .
\end{aligned}
$$

With respect to the behavior of functions $\psi_{k j}(x), k=1,2, j=$ $n_{1}+1, \ldots, n$, the mapping $\pi$ is continuous. From the definition of the mapping $\pi$, we get that $S \cap \Omega_{e}^{0}$ is a retract of $\Omega_{e}^{0}$ and, furthermore, $S$ is a compact set.

By seeing Corollary 3.1, [18, page 282] of Ważewski's theorem [18, Theorem 3.1, page 282] there exists a solution of system (2) with the initial conditions in the set $S \cap \Omega_{e}^{0}$ and it is contained in $\Omega^{0}$ for $x \in(0, \tilde{x}]$. This solution satisfies (5) since the set $\Omega_{e}^{0}$ is contracted to the initial point for $x \rightarrow 0^{+}$.

As we can change the constants $y_{k}^{0}, k=1, \ldots, n_{1}$ within the inequality $v_{k}\left(\tilde{x}, y_{k}^{0}\right)<0$, we can repeat the above-mentioned construction for every admissible fixed set $\left(y_{1}^{0}, \ldots, y_{n_{1}}^{0}\right)$. Then, there exists a class of solutions depending on $n_{1}$ parameters and lying in $\Omega^{0}$ for $x \in(0, \tilde{x}]$. If $n_{1}=n$, the assertion of theorem remains true as well. The proof is complete.

Remark 6. If $\varphi \in M\left(x_{0}, y_{0}\right)$ is a solution of initial problem (2), (5), then

$$
G_{i}(x, \varphi(x))=-\varphi_{i}^{\prime}(x) g_{i}(x, \varphi(x))+\alpha_{i}(x, \varphi(x)) \equiv 0,
$$

where $x \in\left(0, x_{0}\right]$ and $i=1, \ldots, n$, and

$$
G(x, \varphi(x)) \equiv \theta, \quad x \in\left(0, x_{0}\right] .
$$

Then, condition (3) of Theorem 5 is satisfied and, in this case, we obtain the result on the dimension of the set of initial data generating solutions of initial problem (2), (5).

Denote

$$
\begin{aligned}
& R_{i}(y):=\int_{y}^{y_{0}} g_{i}\left(\varphi_{i}^{-1}(t), \varphi\left(\varphi_{i}^{-1}(t)\right)\right) d t \\
& F_{i}(y, \varepsilon):=\frac{R_{i}(y(1+\varepsilon))}{R_{i}(y)}, \\
& F_{i}(0, \varepsilon):=\lim _{y \rightarrow 0^{+}} F_{i}(y, \varepsilon), \\
& \left(F_{i}\right)_{\varepsilon}^{\prime}(0, \varepsilon):=\lim _{y \rightarrow 0^{+}}\left(F_{i}\right)_{\varepsilon}^{\prime}(y, \varepsilon),
\end{aligned}
$$

$i=1, \ldots, n$, provided that the limits exist and are finite. 
Theorem 7. Let all assumptions of Theorem 5 hold and, moreover, the functions

$$
F_{i}: D^{*}\left(y_{00}, \varepsilon^{*}\right) \longrightarrow[0, \infty), \quad i=1, \ldots, n,
$$

satisfy the following:

(1) $F_{i}(y, \varepsilon)$ is continuous with respect to $y$ and continuously differentiable with respect to $\varepsilon$;

(2) $\left(F_{i}\right)_{\varepsilon}^{\prime}(y, 0) \neq 0$.

Then arbitrary solution $y(x)=\left(y_{1}(x), \ldots, y_{n}(x)\right)$ of initial problem (2), (5) mentioned in Theorem 5 has the asymptotic form

$$
y_{i}(x)=\varphi_{i}(x)(1+o(1)), \quad x \longrightarrow 0^{+}, i=1, \ldots, n .
$$

Proof. From the proof of Theorem 5, it follows that, for coordinates $y_{i}(x), i=1, \ldots, n$, of the solution $y(x)$ of initial problem (2), (5), the inequalities

$$
\psi_{1 i}(x)<y_{i}(x)<\psi_{2 i}(x), \quad i=1, \ldots, n
$$

are valid on an interval $(0, \tilde{x}]$ and we can assume that the inequalities

$$
\psi_{1 i}(x)<\varphi_{i}(x)<\psi_{2 i}(x), \quad i=1, \ldots, n
$$

are valid on $(0, \tilde{x}]$ as well. Thus, to prove (89), it is sufficient to prove that

$$
\lim _{x \rightarrow 0^{+}} \frac{\psi_{2 i}(x)}{\psi_{1 i}(x)}=1, \quad i=1, \ldots, n
$$

Applying L'Hospital's rule to the limits (92), we do not obtain the desired result. Therefore we will apply L'Hospital's rule to the limits

$$
\lim _{x \rightarrow 0^{+}} \frac{R_{i}\left(\psi_{2 i}(x)\right)}{R_{i}\left(\psi_{1 i}(x)\right)}, \quad i=1, \ldots, n .
$$

This is possible because, in view of condition (2) of the theorem, we obviously have

$$
\lim _{x \rightarrow 0^{+}} R_{i}\left(\psi_{k i}(x)\right)=\infty, \quad i=1, \ldots, n, k=1,2 .
$$

Then, we use the auxiliary results for implicit functions. Applying the above-mentioned procedure, we get (for $i \in$ $\{1, \ldots, n\})$

$$
\begin{aligned}
\lim _{x \rightarrow 0^{+}} \frac{R_{i}\left(\psi_{2 i}(x)\right)}{R_{i}\left(\psi_{1 i}(x)\right)} \\
=\lim _{x \rightarrow 0^{+}} \frac{\left[R_{i}\left(\psi_{2 i}(x)\right)\right]^{\prime}}{\left[R_{i}\left(\psi_{1 i}(x)\right)\right]^{\prime}} \\
=\lim _{x \rightarrow 0^{+}} \frac{-g_{i}\left[\varphi_{i}^{-1}\left(\psi_{2 i}(x)\right), \varphi\left(\varphi_{i}^{-1}\left(\psi_{2 i}(x)\right)\right)\right] \psi_{2 i}^{\prime}(x)}{\left.\left.-\psi_{1 i}(x)\right), \varphi\left(\varphi_{i}^{-1}\left(\psi_{1 i}(x)\right)\right)\right] \psi_{1 i}^{\prime}(x)} \\
=\lim _{x \rightarrow 0^{+}} \frac{g_{i}\left[\varphi_{i}^{-1}\left(\psi_{2 i}(x)\right), \varphi\left(\varphi_{i}^{-1}\left(\psi_{2 i}^{-1}(x)\right)\right)\right]}{\left.\left.g_{1 i}(x)\right), \varphi\left(\varphi_{i}^{-1}\left(\psi_{1 i}(x)\right)\right)\right]} \\
\times \frac{\alpha_{i}(x, \varphi(x)) / g_{i}\left[\varphi_{i}^{-1}\left(\psi_{2 i}(x)\right), \varphi\left(\varphi_{i}^{-1}\left(\psi_{2 i}(x)\right)\right)\right]}{\alpha_{i}(x, \varphi(x)) / g_{i}\left[\varphi_{i}^{-1}\left(\psi_{1 i}(x)\right), \varphi\left(\varphi_{i}^{-1}\left(\psi_{1 i}(x)\right)\right)\right]} \\
=\lim _{x \rightarrow 0^{+}} \frac{g_{i}\left[\varphi_{i}^{-1}\left(\psi_{2 i}(x)\right), \varphi\left(\varphi_{i}^{-1}\left(\psi_{2 i}(x)\right), \varphi\left(\varphi_{i}^{-1}\left(\psi_{2 i}(x)\right)\right)\right]\right.}{\left.\left.g_{i}\right)\right]} \\
\times \frac{g_{i}\left[\varphi_{i}^{-1}\left(\psi_{1 i}(x)\right), \varphi\left(\varphi_{i}^{-1}\left(\psi_{1 i}(x)\right)\right)\right]}{g_{i}\left[\varphi_{i}^{-1}\left(\psi_{1 i}(x)\right), \varphi\left(\varphi_{i}^{-1}\left(\psi_{1 i}(x)\right)\right)\right]} \\
\alpha_{i}(x, \varphi(x))
\end{aligned}
$$

Hence it follows that there exists a sufficiently small constant $\varepsilon_{i}>0$ such that the inequalities

$$
\begin{array}{r}
R_{i}\left(\psi_{1 i}(x)\right)\left(1-\varepsilon_{i}\right)<R_{i}\left(\psi_{2 i}(x)\right)<R_{i}\left(\psi_{1 i}(x)\right)\left(1+\varepsilon_{i}\right), \\
i=1, \ldots, n,
\end{array}
$$

hold on some interval $\left(0, x^{*}\right], 0<x^{*} \leq \tilde{x}$, where $x^{*}$ is a sufficiently small constant. Applying Lemma 3 and Corollary 4 (formula (27)) we show that inequalities (96) can be written as

$$
\begin{array}{r}
R_{i}\left[\psi_{1 i}(x)\left(1+\varepsilon_{i}^{2}\left(\psi_{1 i}(x), \varepsilon_{i}\right)\right)\right] \\
<R_{i}\left(\psi_{2 i}(x)\right)<R_{i}\left[\psi_{1 i}(x)\left(1-\varepsilon_{i}^{1}\left(\psi_{1 i}(x), \varepsilon_{i}\right)\right)\right], \\
i=1, \ldots, n,
\end{array}
$$

where $\varepsilon_{i}^{j}\left(\psi_{1 i}(x), \varepsilon_{i}\right), j=1,2$, are constants depending on $\psi_{1 i}(x)$ and $\varepsilon_{i}$ such that

$$
0<\varepsilon_{i}^{j}\left(\psi_{1 i}(x), \varepsilon_{i}\right), \quad \lim _{\varepsilon_{i} \rightarrow 0^{+}} \varepsilon_{i}^{j}\left(\psi_{1 i}(x), \varepsilon_{i}\right)=0, \quad j=1,2 .
$$


In Lemma 3 we set, for every fixed index $i=1, \ldots, n$,

$$
\begin{gathered}
R(y):=R_{i}(y)=\int_{y}^{y_{0}} g_{i}\left(\varphi_{i}^{-1}(t), \varphi\left(\varphi_{i}^{-1}(t)\right)\right) d t, \\
F(y, \varepsilon):=F_{i}(y, \varepsilon)=\frac{R_{i}(y(1+\varepsilon))}{R_{i}(y)} .
\end{gathered}
$$

Then, we have $R:\left(0, y_{0}\right) \rightarrow(0, \infty), F: D^{*}\left(y_{00}, \varepsilon^{*}\right) \rightarrow$ $[0, \infty)$ and we have the following:

(a) $R(y)$ is continuously differentiable, $R^{\prime}(y)=$ $-g_{i}\left(\varphi_{i}^{-1}(y), \varphi\left(\varphi_{i}^{-1}(y)\right)\right)<0$

(b) $F(y, \varepsilon)$ is continuous with respect to $y$ and continuously differentiable with respect to $\varepsilon$;

(c) $F_{\varepsilon}^{\prime}(y, 0) \neq 0$ by condition (2) of the theorem.

Hence, Lemma 3 holds. By Corollary 4, we can write inequalities (96) in the form of (97). From (97), we get

$$
\begin{aligned}
\psi_{1 i}(x) & \left(1-\varepsilon_{i}^{1}\left(\psi_{1 i}(x), \varepsilon_{i}\right)\right) \\
< & \psi_{2 i}(x)<\psi_{1 i}(x)\left(1+\varepsilon_{i}^{2}\left(\psi_{1 i}(x), \varepsilon_{i}\right)\right)
\end{aligned}
$$

for $\left(0, x^{*}\right]$. The last inequalities are equivalent to

$$
\lim _{x \rightarrow+0} \frac{\psi_{2 i}(x)}{\psi_{1 i}(x)}=1, \quad i=1, \ldots, n .
$$

The proof is complete.

Example 8. Consider the following simple particular case of initial problem (2), (5):

$$
\begin{gathered}
x^{2}\left(1+x^{2} y^{6}\right) y^{\prime}=y^{2}\left(1+x y^{7}\right), \\
y\left(0^{+}\right)=0 .
\end{gathered}
$$

This problem is a singular one. To apply Theorem 5 , we rewrite (102), (103) as

$$
\begin{gathered}
\frac{1}{y^{2}} y^{\prime}=\frac{1+x y^{7}}{x^{2}\left(1+x^{2} y^{6}\right)}, \\
y\left(0^{+}\right)=0 .
\end{gathered}
$$

Set $n=1$ (below we omit this index since we deal with a scalar problem), $\varphi(x)=x$, and

$$
g(x, y)=\frac{1}{y^{2}}, \quad \alpha(x, y)=\frac{1+x y^{7}}{x^{2}\left(1+x^{2} y^{6}\right)} .
$$

Without loss of generality, we assume that $x_{0}$ and $y_{0}$ in definition of $D_{1}$ are positive and sufficiently small (from Definition (1), property (4), it follows $x_{0}<y_{0}$ ). Condition (1), obviously holds. Condition (2), is valid as well because

$$
\begin{gathered}
\int_{0^{+}} g(x, \varphi(x)) \varphi^{\prime}(x) d x=\int_{0^{+}} \frac{1}{x^{2}} d x=\infty, \\
\int_{0^{+}} \alpha(x, \varphi(x)) d x=\int_{0^{+}} \frac{1+x^{8}}{x^{2}\left(1+x^{8}\right)} d x=\int_{0^{+}} \frac{1}{x^{2}} d x=\infty .
\end{gathered}
$$

Since $\varphi$ is a solution of (104), we have $G(x, \varphi(x)) \equiv 0$ and Condition (3) holds (see Remark 6 as well). Next,

$$
\begin{aligned}
W(x, y) & \\
& =g\left(\varphi^{-1}(y), \varphi\left(\varphi^{-1}(y)\right)\right) \frac{\alpha(x, y)}{g(x, y)}-\alpha(x, \varphi(x)) \\
& =g(y, y) \frac{\alpha(x, y)}{g(x, y)}-\alpha(x, x) \\
& =\frac{1}{y^{2}} \frac{\left(1+x y^{7}\right) /\left(x^{2}\left(1+x^{2} y^{6}\right)\right)}{1 / y^{2}}-\frac{1}{x^{2}} \\
& =\frac{y^{6}(y-x)}{x\left(1+x^{2} y^{6}\right)} .
\end{aligned}
$$

Set $n_{1}=1$ (i.e., conditions (4c) and (4d) are omitted). For $x$ and $y$ sufficiently small and positive, it is easy to see that $W(x, y)>0$ if $y>x$ and $W(x, y)<0$ if $y<x$. Hence, conditions (4a) and (4b) hold. According to Theorem 5, problem (102), (103) has at least one-parametric class of solutions.

Now we apply Theorem 7 . We get

$$
\begin{aligned}
R(y) & =\int_{y}^{y_{0}} g\left(\varphi^{-1}(t), \varphi\left(\varphi^{-1}(t)\right)\right) d t \\
& =\int_{y}^{y_{0}} g(t, t) d t=\int_{y}^{y_{0}} \frac{1}{t^{2}} d t=\frac{1}{y}-\frac{1}{y_{0}}, \\
F(y, \varepsilon) & =\frac{R(y(1+\varepsilon))}{R(y)}=\frac{1 / y(1+\varepsilon)-1 / y_{0}}{1 / y-1 / y_{0}} .
\end{aligned}
$$

Now we see that condition (1) holds since $F$ is continuous with respect to $y$ and continuously differentiable with respect to $\varepsilon$ for $(y, \varepsilon) \in D^{*}\left(y_{00}, \varepsilon^{*}\right)$. The values $F(0, \varepsilon)$ and $F_{\varepsilon}^{\prime}(0, \varepsilon)$ are computed by above given formulas. Moreover, as

$$
\frac{\partial F}{\partial \varepsilon}(y, 0)=\left.\frac{-1 / y(1+\varepsilon)^{2}}{1 / y-1 / y_{0}}\right|_{\varepsilon=0}=\frac{y_{0}}{y-y_{0}} \neq 0
$$

for $y \in\left(0, y_{00}\right]$ with $y_{00}<y_{0}$ and

$$
\begin{aligned}
\frac{\partial F}{\partial \varepsilon}(0,0) & =\lim _{y \rightarrow 0^{+}} \frac{\partial F}{\partial \varepsilon}(y, 0)=-\lim _{y \rightarrow 0^{+}} \frac{1 / y}{1 / y-1 / y_{0}} \\
& =\lim _{y \rightarrow 0^{+}} \frac{y_{0}}{y-y_{0}}=-1 \neq 0 .
\end{aligned}
$$

condition (2) holds too. Theorem 7 is valid and every solution of problem (102), (103) mentioned in Theorem 5 satisfies (89); that is,

$$
y(x)=x(1+o(1))
$$

for $x \rightarrow 0^{+}$.

Finally, we remark that, instead of (102), the same investigation can be performed, for example, for

$$
x^{2}\left(1+x^{2} y^{6}\right) y^{\prime}=y^{2}\left(1+x y^{7}+y^{20}\right),
$$


where the function $\varphi$ is no longer a solution and condition (3) of Theorem 5 holds (the moment $x_{00}$ can be sufficiently small).

\section{Generalization}

The following theorem improves Theorems 5 and 7. In particular, comparing assumptions of Theorems 5 and 7 with Theorem 9, we see that the corresponding assumptions in Theorem 9 are assumed to be valid on some sets that are reductions of sets used in Theorems 5 and 7.

For given constants $C_{i}^{*}>1$ and $0<C_{i}^{* *}<1, i=1, \ldots, n$, and for $\varphi \in M\left(x_{0}, y_{0}\right)$, define sets

$$
\begin{aligned}
N^{\varphi_{i}}\left(C_{i}^{*}\right):= & \left\{(x, y):(x, y) \in D_{n}, \varphi_{i}(x)<y_{i}<C_{i}^{*} \varphi_{i}(x)\right\}, \\
N_{\varphi_{i}}\left(C_{i}^{* *}\right):= & \left\{(x, y):(x, y) \in D_{n}, C_{i}^{* *} \varphi_{i}(x)<y_{i}<\varphi_{i}(x)\right\}, \\
N:= & \left\{(x, y):(x, y) \in D_{n},\right. \\
& \left.C_{i}^{* *} \varphi_{i}(x)<y_{i}<C_{i}^{*} \varphi_{i}(x), i=1, \ldots, n\right\} .
\end{aligned}
$$

Theorem 9. Let, for some constants $C_{i}^{*}, C_{i}^{* *}, C_{i}^{*}>1,0<$ $C_{i}^{* *}<1, i=1, \ldots, n$, and some function $\varphi \in M\left(x_{0}, y_{0}\right)$, the following conditions be fulfilled.

(a) Consider $g_{i}(x, y) \in C(N), \alpha_{i}(x, y) \in C(N), i=$ $1, \ldots, n$.

(b) Assumptions (1)-(3) of Theorem 5 hold.

(c) For $i=1, \ldots, n_{1}$,

$$
\begin{array}{ll}
W_{i}(x, y)>0 & \text { if }(x, y) \in N^{\varphi_{i}}\left(C_{i}^{*}\right) \cap N, \\
W_{i}(x, y)<0 & \text { if }(x, y) \in N_{\varphi_{i}}\left(C_{i}^{* *}\right) \cap N .
\end{array}
$$

We omit this assumption if $n_{1}=0$.

(d) For $i=n_{1}+1, \ldots, n$,

$$
\begin{array}{cc}
W_{i}(x, y)<0 & \text { if }(x, y) \in N^{\varphi_{i}}\left(C_{i}^{*}\right) \cap N, \\
W_{i}(x, y)>0 & \text { if }(x, y) \in N_{\varphi_{i}}\left(C_{i}^{* *}\right) \cap N .
\end{array}
$$

We omit this assumption if $n_{1}=n$.

(e) Functions $F_{i}(y, \varepsilon), i=1, \ldots, n$, satisfy the assumptions of Theorem 7.

Then, there exists at least an $n_{1}$-parametric family of solutions

$$
y(x)=\left(y_{1}(x), \ldots, y_{n}(x)\right)
$$

such that

$$
y_{i}(x)=\varphi_{i}(x)(1+o(1)), \quad i=1, \ldots, n,
$$

holds for $x \rightarrow 0^{+}$and $(x, y(x)) \in D_{n}$.

Proof. The proof is omitted since it is similar to the proofs of Theorems 5 and 7.

\section{Acknowledgments}

The first author was supported by the Project FEKT-S-11-2921 of Faculty of Electrical Engineering and Communication, Brno University of Technology. The third author was supported by the Grant no. P201/11/0768 of Czech Grant Agency (Prague). The second author was supported by the Project CZ.1.07/2.3.00/30.0039 of Brno University of Technology. The work of the first and the third authors was partially realized in (Central European Institute of Technology CEITEC) with research infrastructure supported by the Project CZ.1.05/1.1.00/02.0068 financed from European Regional Development Fund.

\section{References}

[1] A. F. Andreev, Singular Points of Differential Equations, Vysheish. Skhola, Minsk, Russia, 1979, Russian.

[2] K. Balla, "Solution of singular boundary value problems for non-linear systems of ordinary differential equations," USSR Computational Mathematics and Mathematical Physics, vol. 20, no. 4, pp. 100-115, 1980.

[3] J. Baštinec, J. Diblík, and Z. Šmarda, "Multipoint singular boundary-value problem for systems of nonlinear differential equations," Boundary Value Problems, vol. 2009, Article ID 137451, 20 pages, 2009.

[4] J. Diblík, "A multidimensional singular boundary value problem of the Cauchy-Nicoletti type," Georgian Mathematical Journal, vol. 4, no. 4, pp. 303-312, 1997.

[5] J. Diblík, "Sufficient conditions for existence of a pencil of O-curves of a first-order singular differential equation," Differentsial'nye Uravneniya, vol. 21, no. 5, pp. 898-899, 1985 (Russian).

[6] J. Diblik, "On the existence of $O$-curves of a singular system of differential equations," Mathematische Nachrichten, vol. 122, pp. 247-258, 1985 (Russian).

[7] J. Diblik, "Asymptotic behavior of solutions of a system of differential equations near a singular point," Akademiya Nauk Ukrainskoĭ SSR, vol. 35, no. 3, pp. 360-363, 1983.

[8] J. Diblík and C. Nowak, "A nonuniqueness criterion for a singular system of two ordinary differential equations," Nonlinear Analysis. Theory, Methods \& Applications, vol. 64, no. 4, pp. 637656, 2006.

[9] J. Diblík and M. Růžičková, "Existence of positive solutions of a singular initial problem for a nonlinear system of differential equations," The Rocky Mountain Journal of Mathematics, vol. 34, no. 3, pp. 923-944, 2004.

[10] J. Diblík and M. Růžičková, "Inequalities for solutions of singular initial problems for Carathéodory systems via Ważewski's principle," Nonlinear Analysis. Theory, Methods \& Applications, vol. 69, no. 12, pp. 4482-4495, 2008.

[11] J. Kalas, "Nonuniqueness theorem for a singular Cauchy problem," Georgian Mathematical Journal, vol. 7, no. 2, pp. 317-327, 2000.

[12] S. K. Norkin, "Local and asymptotic whirlpools for integral $O$ manifolds," Akademiya Nauk Ukrainskoĭ SSR, vol. 37, no. 3, pp. 317-322, 1985.

[13] I. T. Kiguradze, Some singular boundary value problems for ordinary differential equations, Izdat. Tbilis. Univ., Tbilisi, Georgia, 1975, Russian. 
[14] I. T. Kiguradze, "Cauchy problem for singular systems of ordinary differential equations," Differentsial'nye Uravneniya, vol. 1, no. 10, pp. 1271-1291, 1965.

[15] V. A. Chechyk, "Investigation of systems of ordinary differential equations with a singularity," Trudy Moskovskogo Matematičeskogo Obščestva, vol. 8, pp. 155-198, 1959 (Russian).

[16] N. B. Konyukhova, "Singular Cauchy problems for systems of ordinary differential equations," Akademiya Nauk SSSR, vol. 23, pp. 72-82, 1983.

[17] T. Ważewski, "Sur un principe topologique de l'examen de l'allure asymptotique des intégrales des équations différentielles ordinaires," Annales de la Société Polonaise de Mathématique, vol. 20, pp. 279-313, 1947.

[18] P. Hartman, Ordinary Differential Equations, SIAM, 2nd edition, 2002.

[19] R. Srzednicki, "Ważewski method and Conley index," in Handbook of Differential Equations, A. Caňada, P. Drábek, and A. Fonda, Eds., vol. 1, pp. 591-684, Elsevier, Amsterdam, The Netherlands, 2004.

[20] K. Borsuk, Theory of Retracts, Mir Publishers, Moscow, Russia, 1971, Russian. 


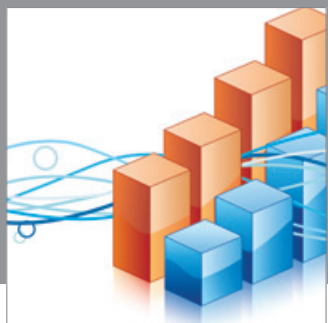

Advances in

Operations Research

mansans

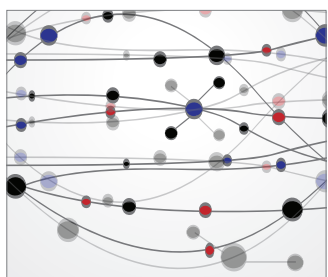

The Scientific World Journal
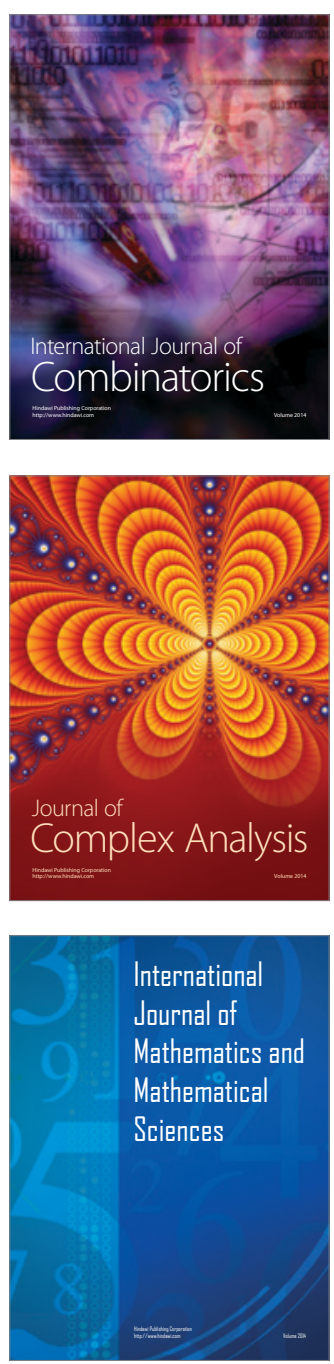
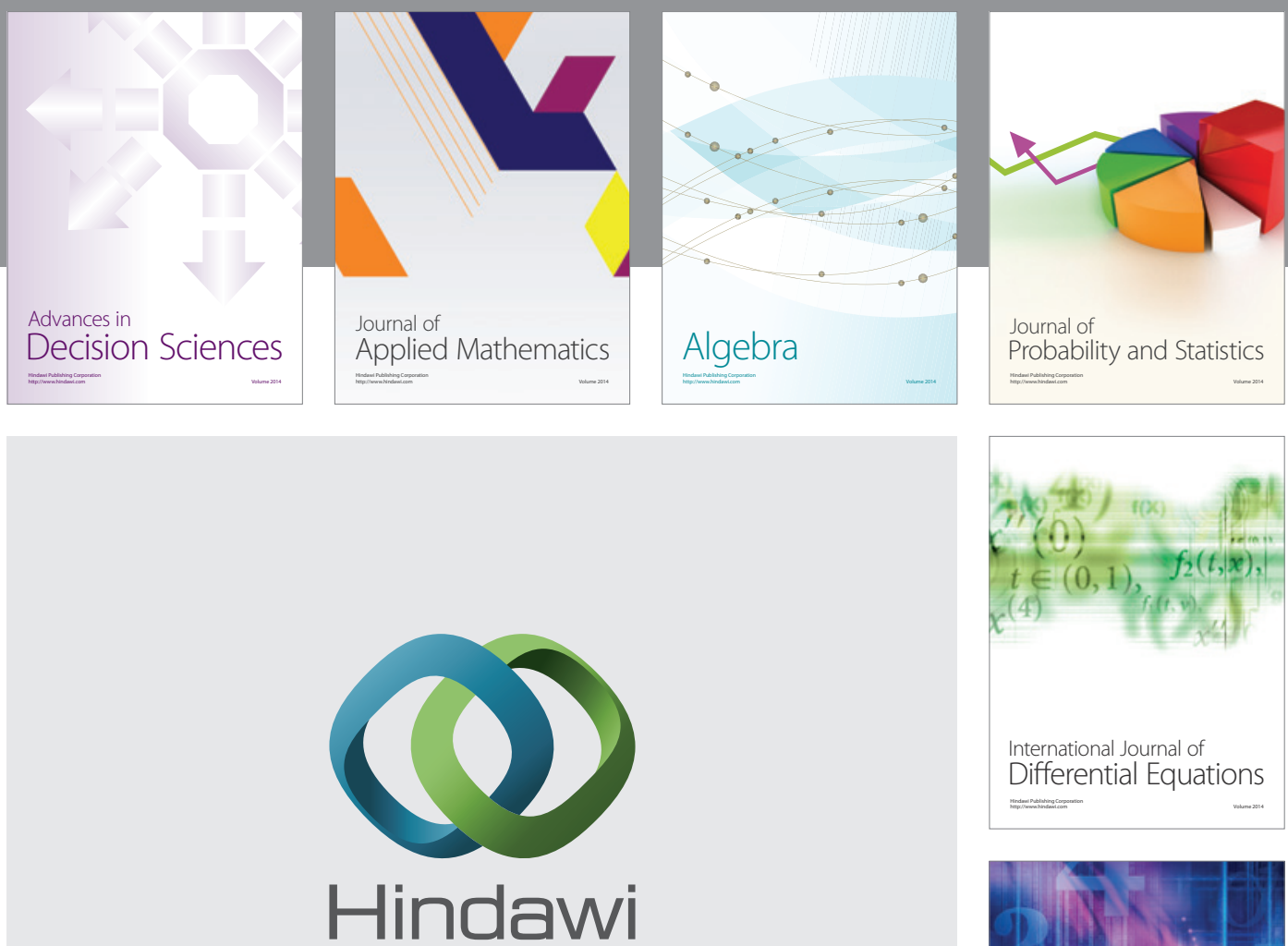

Submit your manuscripts at http://www.hindawi.com
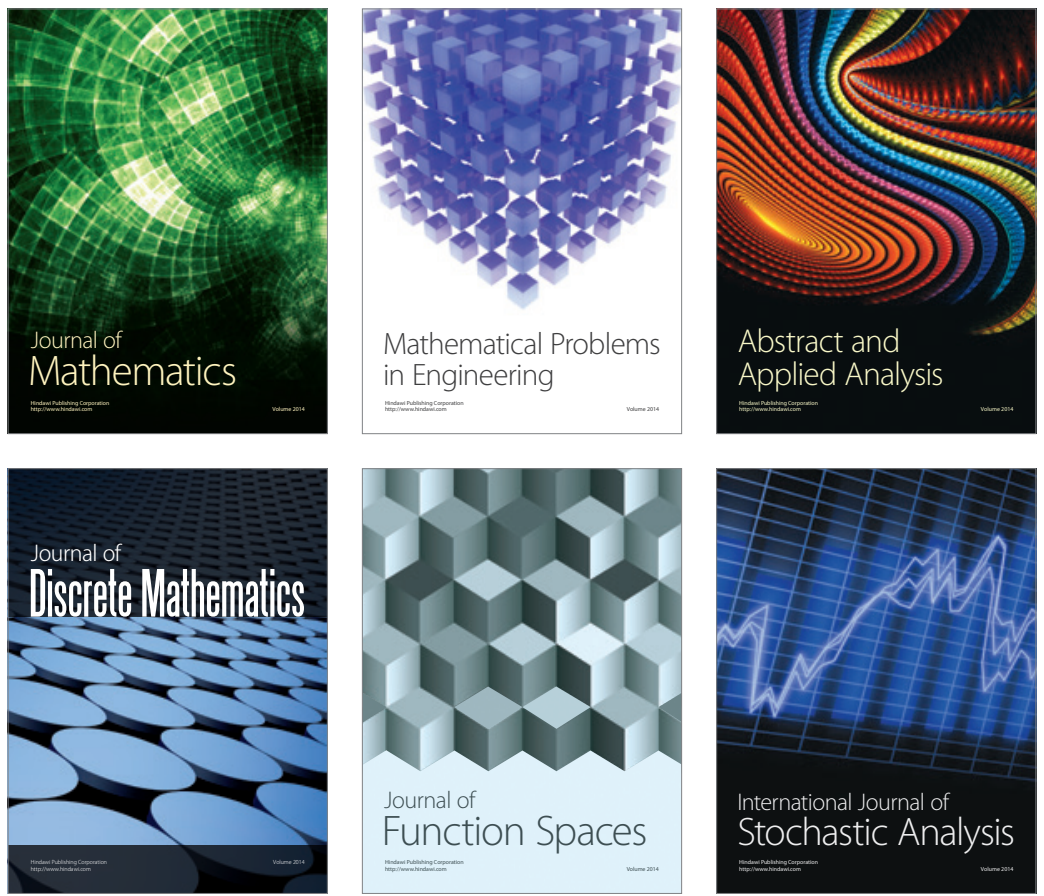

Journal of

Function Spaces

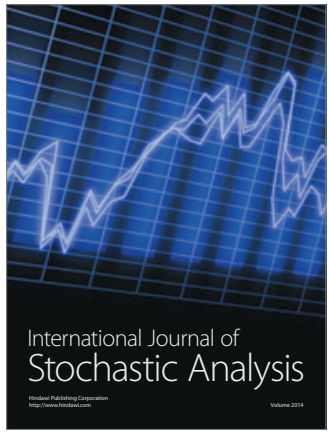

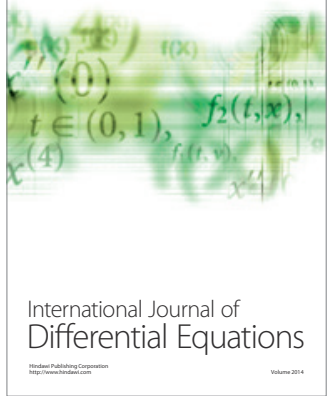
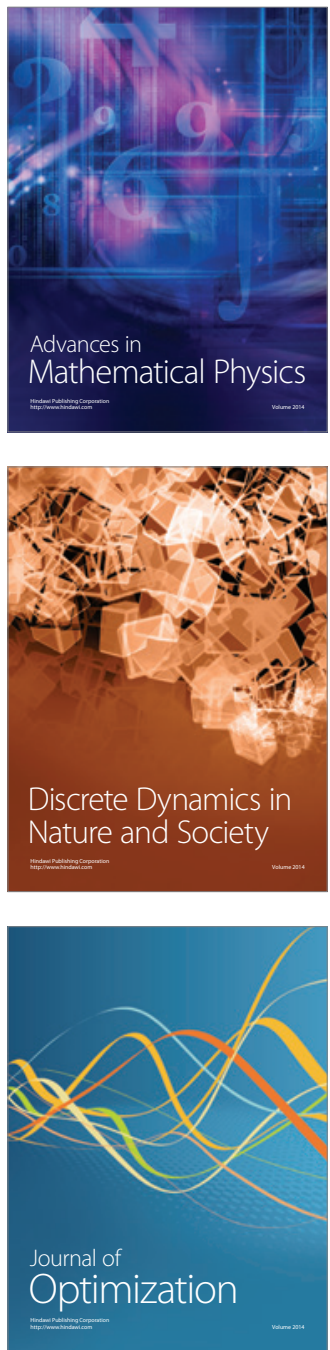